\title{
Trois écrivains au café : Interactions et socialité en littérature québécoise contemporaine
}

\author{
David Bélanger \\ Université du Québec à Montréal
}

L'écrivain au café : l'image agace, trop convenue. Mythifiée, elle contient toutes les constituantes du métier d'écrire. La solitude à sa table, mise en contraste par la présence des autres, le caractère public de l'écriture, mais l'individualité de la tâche. C'est toutefois sur le plan des socialités littéraires que le café a été davantage traité dans la critique, et là surtout agissent les poncifs et les idées reçues. L'écrivain au café paraît intégré dans un ferment social, contrairement, par exemple, à l'écrivain de salon; plus démocratique, capable de générer des groupes et des 
écoles, le café, comme l'écrit Vincent Laisney, serait donc «un instrument idéal de cohésion au plan social, et de cohérence au plan esthétique » $(2010, \S 3)$. On y écrit et on y vit la chose littéraire.

Ce lieu commun agit sur le plan des représentations culturelles. Le Flore de Sartre ou, moins café que bar - mais l'histoire littéraire fait-elle de telles distinctions? - le Ritz d'Hemingway, le Café Voltaire des Dada ou celui de la Place Blanche des surréalistes, voilà autant de mythes sur le fond desquels se structure l'imaginaire de l'écriture et de la scène littéraire. Tous sis en Europe - presque tous à Paris -, tous ancrés dans une certaine modernité, ces cafés paraissent toutefois difficilement exportables dans l'espace et dans le temps, comme un monde clos, figé, lointain, difficile à investir, mais lourd de tradition. L'analyse de la représentation du café dans le roman québécois contemporain impose donc une certaine prudence.

Ainsi, deux pas de côtés, deux questions, animeront cet article, et j'entends les expliciter avant d'avancer plus avant dans mon sujet : quelle forme cet imaginaire du café de l'écrivain prendelle maintenant dans les fictions, en ce que le mythe, ancien, ne peut être simplement réaffirmé et en ce que l'usure du mythe n'invite d'aucune manière sa reconduction? Pour mieux dire : quel statut aura le café de l'écrivain dans la fiction contemporaine, comment s'effectuera sa médiatisation du lieu commun ? Plus ennuyeux peutêtre, je ne pourrai faire abstraction du statut du café en littérature québécoise; le mythe du café est le mythe d'un autre, d'une certaine manière, puisque dans l'histoire du Québec, très peu de cercles s'y sont formés, très peu d'écrivains y ont écrit - c'est du moins ainsi qu'on se le raconte. 
En effet, difficile au Québec de trouver un café frappé par le mythe littéraire; le café Ayotte du groupe des "Six éponges » reste beaucoup moins célèbre que le Château Ramezay, où Nelligan déclama sa « Romance du vin », ou l'arrière-boutique de la librairie d'Octave Crémazie, où la communauté lettrée de Québec faisait "école». On s'étonnera d'apprendre que SaintDenys Garneau écrivit une grande part de son Regards et jeux dans l'espace dans les cafés et les restaurants de Montréal (Biron, 2015, p. 248-251) alors qu'on imagine plus harmonieu-sement sa solitude dévorante dans le « manoir » de Sainte-Catherine-dela-Jacques-Cartier. À ce chapitre, les mots de la préface de Louis Dantin aux poésies d'Émile Nelligan donnent une juste idée des connotations en place :

Notre Canada est assez pauvre en gloires littéraires pour que nous recueillions précieusement les moindres miettes de génie tombées de notre table. Et pourtant, plus que d'autres, nous sommes ingrats envers nos gloires. Grâce à cette illusion d'optique qui fait voir merveilleux tout ce qui est lointain, les talents les plus discutables trouvent chez nous des admirateurs et des disciples, pourvu que leurs écritures soient estampillées de Charpentier ou de Lemerre. Mais nous répugnons à l'idée qu'un bon garçon que nous coudoyons tous les jours, avec qui nous prenons la goutte au petit Windsor, dont nous connaissons les faiblesses, les travers, voire les douces manies, porte en lui l'étoffe d'un Rodenbach ou d'un Rollinat. La camaraderie tue chez nous l'admiration. (1997 [1904], p. 67)

La camaraderie du café tue l'admiration, empêche la reconnaissance; la proximité du café, plutôt que de faire réseau, cohésion sociale et cohérence esthétique, rend suspect. Cette connotation du café paraît porter la représentation de l'écrivain dans le roman québécois; l'écriture s’y épanouira davantage 
dans le lieu fermé, chambre, cuisine, clinique psychiatrique ${ }^{1}$. Nous en revenons ainsi à l'hypothèse bien connue de Michel Biron selon laquelle «quelle que soit sa forme, la solitude de l'écrivain constitue peut-être le plus ancien et le plus profond motif de complainte de la littérature québécoise » (2000, p. 37). Car, écrit-il, l'écrivain québécois des premiers temps ne trouve pas dans sa société d'autres écrivains à qui se mesurer; il ne peut donc appartenir à quelque groupe et, par là, ajouterai-je, il ne peut vivre le café comme idéal de cohésion, car sans cohésion, évidemment, plongé dans la plus pure solitude, nul café. Biron montre bien, en fait, que c'est la coupure entre l'élite lettrée et le reste de la population, au Québec, qui rend «l'identité de groupe », lettrée et intellectuelle,

difficile à revendiquer, car tout en fournissant un cadre de référence positivement connoté, [une telle identité de groupe] isole l'intellectuel québécois à l'intérieur de la société et contribue à renforcer sa liminarité. [...] L'écrivain ne s'oppose pas à un autre écrivain, mais à celui qui ne sait pas écrire et qui incarne paradoxalement une figure privilégiée de la modernité québécoise. (2000, p. 32-33)

Ces hypothèses concernant la littérature québécoise, qui résonnent d'ailleurs fortement avec les constats d'André Belleau sur le romancier fictif des années $1940-1960^{2}$, nous indiquent

\footnotetext{
${ }^{1}$ François Galarneau, dans Salut Galarneau ! de Jacques Godbout (1965), écrit dans sa cuisine, et sa maison s'isole du reste du monde alors qu'il fantasme sur la possibilité de vivre et d'écrire - c'est le fameux « vécrire ». Les personnages de Gérard Bessette écrivent dans leur chambre, bien qu'ils errent également dans les tavernes, où ils se gardent toutefois d'écrire ou même de parler de littérature : dans La Bagarre (1958) et Le Libraire (1960). On aura reconnu l'écriture du narrateur de Prochain Épisode (1966) d'Hubert Aquin dans une clinique psychiatrique.

${ }^{2}$ Ce constat sourd durant tout l'essai de Belleau et dans sa théorisation du « conflit de codes » également, mais s'explicite à la fin du Romancier fictif en ces termes : «Ce qui fait problème dans tant de nos romans à personnage-écrivain,
} 
une tradition dans laquelle l'absence du café comme l'absence d'un monde littéraire plein, décomplexé, marche-raient ensemble dans l'histoire culturelle québécoise. Les écrivains contemporains se trouvent donc devant cette double tradition, celle d'un lieu commun lointain et emprunté et celle d'une rareté étonnante soulignant l'inadéquation entre la vie culturelle de l'individu et l'absence de reconnaissance de la culture dans les premiers temps de la société canadienne-française. L'objet du présent article sera précisément d'observer ce que signifie le café dans sa représentation de la littérature; en regard des traditions contradictoires, de l'usure du mythe, mais aussi du symbole même que constitue écrire en public, il s'agira de comprendre ce que dit le café représenté de la littérature. En analysant la représentation du romancier dans le café dans trois œuvres québécoises contemporaines, je me propose - et je m'en tiendrai ici à cette seule hypothèse, qu'il conviendra de préciser en fin de parcours - d'analyser le café comme médiateur entre la société et la littérature. Sciemment, j'avancerai par étape, œuvre par œuvre, étant entendu que chaque représentation structure un autre discours sur la littérature, discours qu'il conviendra de rapailler en conclusion. Chez Patrice Lessard, François Blais et Mylène Bouchard, ainsi, j'entends mener une enquête sur un lieu commun, trop commun, de la littérature.

\section{Désintégré : le café chez Patrice Lessard}

ce n'est pas l'anti-intellectualisme, un parti pris contre la culture (on n'en finit plus de dénombrer les références à la littérature et aux arts chez Harvey, Lemelin, Charbonneau, Hamel, Simard, Bessette, Godbout), c'est la répartition même des signes, le fait que la culture, peu importe sa position d'ailleurs variable dans l'une ou l'autre série, se trouve constamment opposée à quelque chose. [...] presque toujours, l'auteur-narrateur ne réussit à se projeter comme auteur-personnage que dans la dualité, par le dédoublement de ce dernier en deux instances contrastantes. » (1999 [1980], p. 205) 
Patrice Lessard, écrivain mis en scène dans le dernier volet de la trilogie lisboète de Patrice Lessard, est fort désespéré de la fermeture de ses cafés préférés à Lisbonne, dans ces cafés où il avait ses habitudes : «si je ne pouvais plus écrire à la Casa do Alentejo ni à la Leitaria, qu'est-ce que je ferais à Lisbonne? » (2014, p. 36) La question revêt quelque chose d'existentiel, comme si ces séjours d'écriture qui ont vu naître les œuvres de Lessard étaient avant tout pétris de ce rapport au lieu, double ici : Lisbonne, d'une part, car la trilogie lisboète de Lessard, comprend-on dans cet ultime volume autofictif, demandait à ce que l'auteur vive la ville, mais surtout, d'autre part, une Lisbonne publique, dont le café constitue l'interface privilégiée. C'est « à la recherche d'un lieu où écrire » (2014, p. 37) que se lancent alors le romancier et sa compagne, Clara; devant le luxueux Hotel Avenida Palace, les pas de Lessard bifurqueront, attirés par ses halls ouverts et son faste de pacotille : «Tu ne vas tout de même pas écrire dans un hôtel! " , s'étonne Clara. Dans ces halls, on ne lit pas les philosophes ou les écrivains dans le vent, on ne lit rien de culturel; le Financial Times et le Diário Ecónomico seuls font l'objet de quelque attention. Le romancier ne trouvera pas là le lieu de son écriture; il sera d'ailleurs invité à quitter l'endroit. C'est plutôt dans un petit troquet poussiéreux qu'il élira domicile. Dans une petite salle au fond d'un café aux murs « d'un bleu poudre jauni par le tabac, la saleté, le temps ", l'écrivainnarrateur se trouve à son aise : «C'était parfait» (2014, p. 4445), s'exclame-t-il. Ce café, sans que l'écrivain n'y appartienne en propre, l'intègre : « vous êtes Portugais ? Je n'ai pas vraiment une tête de Portugais et je parle la langue, alors on me pose souvent la question, je répondis, Non, je ne suis pas portugais, et lui, Vous parlez très bien portugais ! je croyais que vous étiez portugais ! » 
(2014, p. 45) Patrice Lessard s'intégrera alors à la faune qu'il observe, à la ville qu'il décrit, il la verra de l'intérieur. Ainsi, au fond de la salle du café, il épiera un curieux manège interlope, fait de sacs dissimulés dans des vases de fleurs et retirés un peu plus tard. L'écrivain, dans ce café, participera au monde et à son action.

Ce que je voulais souligner par cette rapide présentation de L'Enterrement de la sardine tient en peu de mots : le café, comme lieu d'écriture, appelle avant tout chez Lessard la notion d'intégration. L'écrivain s'y intègre à la faune en place, on le perçoit comme portugais, il donne un coup de main au tenancier de l'établissement, préparant des cocktails, jouant l'interprète. Mais cette intégration de l'écrivain dans le ferment social suggère en elle-même le décalage que tente de fuir l'écriture; s'organise en fait une dialectique du café entre ce lieu commun littéral - le lieu de tous que ce troquet représente - et littéraire le stéréotype de l'écrivain, que les cafés maintenant fermés symbolisent ${ }^{3}$. Cette dialectique consiste en une indécision, une négociation dont le lieu d'écriture, le café, devient le révélateur. Dominique Maingueneau, dans ses ouvrages des vingt dernières années, répète le statut fondamental d'une telle dialectique en littérature; il la nomme paratopie :

Sans « localisation » il n'y a pas d'institutions permettant de donner un statut à la production et la consommation des œuvres, mais sans « dé-localisation » il n'y a pas à proprement parler de création. L'appartenance au "champ» littéraire n'est pas l'absence de tout lieu, mais plutôt une difficile négociation, dans le discours même, entre le lieu et le non-lieu : cette localisation parasitaire, cette appartenance paradoxale qui vit de

\footnotetext{
${ }^{3}$ Le Leitaria, ainsi, signifie en portugais laiterie. Le terme rappelle toutefois l'activité littéraire par excellence : Leitura (lecture).
} 
l'impossibilité même de définir une véritable appartenance, nous avons proposé de la nommer paratopie. (2006, p. 66-67)

On pourrait dire que la fiction de Patrice Lessard, dès Je suis Sébastien Chevalier, travaille cette antinomique paratopie. En même temps, ses œuvres ne cessent de répéter les limites de ce concept, en dépassant - du moins sur le plan de la représentation de l'écrivain - la notion de champ littéraire ou d'institution littéraire, lui préférant l'autre grand ensemble en regard duquel se situerait l'écrivain: la culture nationale. L'écrivain, pour mieux dire, se situe plutôt vis-à-vis d'une conception partagée de la culture au Québec que par rapport à une pratique de la littérature légitimée, et cela, me semble-t-il, parle beaucoup du statut de la littérature nationale et de l'écrivain dans cet ensemble. Car l'écrivain et l'écriture, comme l'indiquait déjà Michel Biron à propos de la littérature québécoise des premiers temps, ne trouvent pas d'autres écritures et d'autres écrivains auxquels s'opposer; ils ne trouvent que la société dans son ensemble, et cette société prend la forme hégémonique de la culture nationale. La nouvelle «Kultura», dans Je suis Sébastien Chevalier, montre bien comment s'organise cette mauvaise humeur ${ }^{4}$ de l'écrivain face à sa société : en présentant un docteur en géologie polonaise, échoué dans le quartier Saint-Laurent à Montréal, plongé dans une écoute aphasique de la télévision et de la campagne référendaire de 1995 qu'elle colporte, cette nouvelle souligne la fragilité de l'ensemble social et de son expression que serait la culture québécoise. Devant une déclaration de Lucien Bouchard

\footnotetext{
${ }^{4}$ Je paraphrase ici Michel Biron, qui note que « l'écrivain [québécois] ne bute contre aucun autre écrivain, son texte ne heurte aucun autre texte avant de s'abîmer dans le néant de la société d'épiciers. [...] Il n'en reste qu'une sorte de mauvaise humeur dirigée de manière diffuse contre la société dans son ensemble. » (2000, p. 29)
} 
sur CTV, « quelque chose de plus ou moins insignifiant à propos de la "culture" » (2009, p. 21), le docteur s'esclaffe, un rire qui brise le silence dans lequel il était plongé depuis dix ans pour enfin s'exclamer : "But what is culture ? » $(2009$, p. 23) Au-delà du discours vaguement antinationaliste que porte cette nouvelle, nous avons ici une véritable idéologie dans le texte ${ }^{5}$, comme si, toujours chez Lessard, on s'opposait au grand ensemble abstrait (économique, politique, culturel), appelant plutôt une intégration du sujet (dans la culture, dans la littérature). L'abstraction décale - dans son salon -; la vie, la concrétude, intègre - dans un café. Pourtant, et voici un passionnant paradoxe, le discours antinationaliste qui traverse les textes de Lessard fête une sorte de dé-localisation heureuse, alors même que les structures de ses fictions semblent appeler une localisation pleine, manière d'être enfin intégré dans la marche du monde.

Les cafés de Je suis Sébastien Chevalier, ceux notamment qui encadrent le recueil - avec les nouvelles «Je suis Patrice Lessard» et «Je suis Sébastien Chevalier»- présentent ce paradoxe sous la forme d'un narrateur vivant pleinement la culture, capable de la faire sienne, de la posséder, voilà une forte localisation; mais cette culture s'éprouve jusqu'à sa déréalisation, jusqu'à ce que l'intégration de l'écrivain au café se transforme en décalage. Ainsi, le protagoniste de la première nouvelle est hanté par une phrase qu'il répète dans sa tête : «Je suis Patrice Lessard ». Pour fuir ce leitmotiv, il se rend dans un café du Plateau Mont-Royal, l'El Dorado, où il se plonge dans la

\footnotetext{
${ }^{5}$ L'expression, d'André Belleau, se trouve à la fin de son Romancier fictif: à l'idéologie dans le texte, fait du " discours du narrateur et des personnages », Belleau oppose l'idéologie du texte, « liée à la structure même du texte conçu comme ensemble, comme objet artistique » (1999, p. 214).
} 
lecture de la revue Séquence consacrée au cinéaste André Turpin. Or, apparaît à la table d'à côté nul autre que David Lahaye, acteur principal d'Un crabe dans la tête, œuvre-phare d'André Turpin. Le protagoniste ne peut s'empêcher de le saluer, y allant de compliments - "vous êtes un acteur exceptionnel, j'ai vu Un crabe dans la tête et même Nouvelle-France et malgré ce qu'en ont dit les critiques, chapeau ! ». Étonné, David Lahaye ne sait quelle attitude adopter et laisse tomber, tout simplement : « Mais je ne suis pas David Lahaye... » L'individu plaça alors le portrait de Lahaye dans la revue Séquence à côté de son visage et l'évident décalage «frappa [le protagoniste] comme une gifle». Le personnage fuit alors les lieux et réalise, "sous le regard perplexe de son interlocuteur, qu'il n'était pas, lui-même, Patrice Lessard » (2009, p. 8-10). Le phénomène s'avère fort semblable dans la dernière nouvelle du recueil : un comédien se rend dans le café l'El Dorado pour rencontrer un ami metteur en scène. L'attendant, il lit La Méprise de Vladimir Nabokov. Une phrase le hante, toutefois, qu'il murmure à mi-voix: Je suis Sébastien Chevalier. Son voisin de table perd patience et l'apostrophe: «C'est quoi ton hostie de problème ? Veux-tu une claque ? [...] Ça fait dix minutes que tu marmonnes comme un hostie de déficient pis que tu dis mon nom aux huit secondes. [...] Sébastien Chevalier, c'est moi. » Le protagoniste convient aussitôt : "Il était vraiment confus. Il venait de reconnaître Sébastien Chevalier. L'écrivain.» (2009, p. 159-160) Puis la fin de la nouvelle révèle, ajoutant un tour au manège, que le protagoniste se prénomme Patrice, renvoyant au Patrice de la première nouvelle et, incidemment, au Patrice Lessard écrivain.

Ces deux cafés qui reviennent ici et là dans le recueil montrent, ai-je postulé, une incarnation de la culture; en localisant la culture, c'est-à-dire en la déplaçant de son lieu symbolique - le 
star system, les revues, les livres - et en faisant apparaître ceux-ci dans un espace de rencontre, la culture y est vécue. Mais vécue, localisée, elle se décale plutôt qu'elle ne s'intègre. Il convient de regarder de plus près l'impasse à laquelle cette dialectique nous mène. Laurence Côté-Fournier remarque avec justesse, à propos de Je suis Sébastien Chevalier, la constante référence à la métafiction américaine, dont La Vraie Vie de Sebastian Knight de Nabokov constitue un texte canonique ${ }^{6}$. Mais à la différence de cette métafiction, note-t-elle, les

jeux formels [chez Lessard], plutôt que d'ouvrir des abîmes métaphysiques comme chez Nabokov, se situent du côté d'une inquiétante étrangeté aussi ridicule que déstabilisante. Ce ridicule est causé en grande partie par la tension entre art et culture populaire qui traverse le recueil et tue dans l'œuf toute grandiloquence. (2016, p. 35-36)

La métafiction américaine, comme le Nouveau Roman français par ailleurs, témoigne d'une confiance envers les hiérarchies culturelles; chez Lessard, cette confiance n'agit guère, la grandiloquence s'efface et devient farce. Cependant, le jeu des nouvelles liminaires du recueil ne s'avère pas stérile; il souligne bien, grâce à sa double métalepse, le lieu de l'écriture. Patrice Lessard sort de scène, y étant entré contre toute logique, comme David Lahaye sort de scène au terme d'une courte confusion. La scène d'où sortent ces acteurs culturels, c'est le café. Tout se passe comme si cette nouvelle inaugurale servait à désamorcer l'écriture dans ce qu'elle supporte de culturel pour en permettre une incarnation plus libre - libérée de l'insignifiance qu'est la culture. Côté-Fournier termine son

${ }^{6}$ Côté-Fournier, en plus d'expliciter le clin d'œil entre Sebastian Knight et Sébastien Chevalier, montre que certains procédés de Feu pâle du même Nabokov sont intégrés dans le recueil (2016, p. 34-35). 
analyse de Je suis Sébastien Chevalier en écrivant que, pour Lessard, « l'exhibition des poncifs du monde culturel constitue la meilleure façon de montrer qu'il ne saurait $\mathrm{y}$ adhérer pleinement » (2016, p. 36). Il y a de cela dans le lieu commun du café de Lessard. D'un côté, l'écrivain écrit à sa table sans être reconnu, il est dérangé dans son stéréotype; d'un autre, il s'efface, disparaît en quittant le café, laissant la place à l'action, au texte, aux anecdotes quotidiennes plutôt qu'aux questions culturelles. Qu'on me permette, pour fermer la porte sur le café de Patrice Lessard, un dernier exemple. Tirée d'un collectif en mémoire du Printemps étudiant de 2012, la nouvelle «Comme les Hell's » se passe dans un café de Paris. L'écrivain est appelé à raconter la « révolution » en marche au Québec à une Française curieuse : «enfin, ce n'est pas vraiment une révolution, trop de gens trop cons pour comprendre» (2012, p. 94), précise-t-il. Mais être à Paris durant les événements qui se déroulent au Québec pourrait lui donner l'apparence du fuyard : l'écrivain se justifie aussitôt, racontant du même coup sa traversée de Paris vers le Café Nord-Sud où se joue le cœur du texte, d'où se développe l'énonciation :

J'aime la vieille et bringuebalante et crissante ligne 4 avec ses odeurs d'aisselle, de vieux fromage et de caoutchouc brûlé, l'impression que tous les drames quotidiens s'y trouvent en germe, les esclandres, les ronds de jambe et les pitreries qui me passionnent, me poussent à inventer, des histoires, des mondes où fuir, je me suis sauvé, pensai-je alors [...] je ne me suis pas vraiment sauvé, en fait, je veux dire, cette courte escapade à Paris était prévue depuis un certain temps, officiellement je ne fuyais pas la grève ou les conséquences de la grève ou la bêtise ambiante, cela dit, la fuite, c'est un peu dans ma manière, alors ça tombait bien, disons, d'un point de vue esthétique[.] (2012, p. 89) 
Tout, dans ce court passage, permet d'expliciter la dialectique qui agit au cœur du café de Lessard. D’un côté, nous avons les «drames quotidiens» - "C'est dans les drames anodins et quotidiens que m'apparaît parfois le sens des choses, pas dans les catastrophes nationales » (2012, p. 88), dit-il au début de la nouvelle -, des drames qui offrent des mondes où fuir, où s'intégrer, aussi bien dire des fictions, des avenues anodines sur lesquelles structurer ses textes. Le troquet vaguement interlope, l'El Dorado des imbroglios, voilà des drames anodins, loin des grands ensembles, des catastrophes nationales. D'un autre côté, nous avons une esthétique de la fuite, qui consiste à aborder de biais un réel désincarné, réel économique, politique, culturel, national. C'est dans le déplacement, dans le décalage - depuis Lisbonne, depuis le regard d'un spécialiste polonais en géologie, depuis Paris - que le lieu de l'écriture peut accueillir les discours de la société. Entre cette localisation et cette dé-localisation, une incarnation dans le café et une désintégration hors de la société, nous avons une tension des plus productives. À la fin de cette nouvelle du Printemps étudiant, l'écrivain est rejoint par sa compagne. Il lance, le regard tourné avec révolte sur les événements politiques du Québec, voulant prolonger sa fuite : «Je ne veux pas rentrer, On peut rester si tu veux, proposa-t-elle, elle devait parler du café. Pas moi. » (2012, p. 96) Le café est ce lieu où il façonne ses mondes où fuir, d'où il peut percevoir sans s'investir, participer sans subir, analyser les plus modestes sans pour autant leur appartenir. L'expression de Maingueneau gagne alors en résonance, comme si le café, chez Lessard, en traçait la définition : la paratopie est ce lieu de faille pour l'écrivain qui "n'a pas lieu d'être - aux deux sens de la locution - et qui doit construire le territoire de son œuvre à travers cette faille même » 
(2006, p. 68). Ce territoire est un café, à la manière d'un portail, entre le dedans et le dehors, l'intégration et le décalage.

\section{Liminaire : le café chez François Blais}

Qu'est-ce qu'un café situé sur le Plateau-Mont-Royal, à Montréal, organise dans le discours littéraire qu'un café, disons à GrandMère, ne pourrait organiser ? Voilà l'enjeu au cœur de l'œuvre de François Blais: moins être décalé qu'être liminaire, moins paratopique que marginal. Ce constat de départ devra être nuancé; toutefois, convenons déjà que le discours littéraire nourri dans l'œuvre de Blais revendique son inadéquation avec une scène culturelle centrale. Les romans de Blais mettent tous en scène, plus ou moins, des écrivains, scripteurs au statut changeant, lecteurs à la recherche d'auteurs, personnages pétris de littérature. L'écrivain y prend des formes hybrides et limites, généralement érigé contre l'institution littéraire : il n'écrit que pour lui-même, il écrit pour frauder les instances de légitimation, il sur-joue pompeusement l'habitus lettré. Le café, le bar, le petit restaurant occupent une place importante dans cette gestion du discours littéraire; ainsi, dans Document 1, les deux narrateurs tentent d'écrire un roman afin de mettre la main sur une bourse du Conseil des arts du Canada. Pour ce faire, ils étudient les protocoles d'édition des éditeurs québécois. La question formelle au cœur de ces protocoles est vite ironisée au profit de questions plus essentielles :

Bref, toutes les maisons d'édition ont leurs préférences quant à la présentation des manuscrits qu'ils reçoivent, mais pour ce qui est du contenu, ils laissent ça à notre entière discrétion. (Pourtant, si j'étais éditrice, il me semble que j'aurais plutôt tendance à dire : «Présentez ça comme vous voulez, pourvu que 
ça soit lisible, vous pouvez même y aller au stylo si vous avez une belle main, mais soyez avisé que tout manuscrit relatant les aventures d'un trentenaire noyant sa peine d'amour dans un quelconque bar du Plateau-Mont-Royal sera envoyé directement à la déchiqueteuse. ») (2012, p. 84)

Le bar montréalais devient l'archétype romanesque de la littérature québécoise contre lequel s'oppose le discours des écrivains frauduleux de François Blais; de même, le centre culturel montréalais semble fait de règles factices, rhétoriques avant tout, à côté de l'essence de l'art. Lorsqu'un écrivain légitimé, exilé à Grand-Mère, consent à prêter son identité aux narrateurs afin qu'ils confectionnent une demande de bourse, il consent du même souffle à les aider dans la rédaction du formulaire, faisant s'extasier la narratrice : «Il connaissait les buzzwords qui allument les littéraires et savait créer cette profondeur factice à laquelle se laissent prendre même ceux qui ont vu neiger. » (2012, p. 103) La littérature et ses hiérarchies apparaissent alors simplement arbitraires. Le café agit vite dans cette destitution de l'institution littéraire en ce qu'il cristallise la règle sociale ambiguë dont doivent répondre les personnagesécrivains. Prenons l'exemple de Sébastien Daoust, écrivain reconnu dont s'inspirent les écrivains frauduleux de Document 1. Après deux romans expérimentaux - de la «schnoute expérimentale » (2012, p. 85), commente Tess, la narratrice - et une thèse sur le Temps chez Paul Valéry, il abandonne la littérature : « [p] arce que j'y crois plus » (2012, p. 72), laisse-t-il tomber. Sa désertion détermine son portrait d'écrivain : il est devenu un ouvrier consacré à la construction de bateaux, qui tous les midis visite Tess au restaurant Subway, là où elle lui confectionne un sandwich - « un douze pouces aux boulettes marinara, qu'il accompagne d'un Coke Diet et de biscuits aux noix de Macadam»(2012, p.54). La rencontre entre Tess, 
narratrice qui veut devenir écrivain, et l'écrivain légitime ne peut alors trouver quelque romantisme, la scène ne peut souffrir d'un lyrisme d'écriture, toute fondue dans la confection du sandwich : « quelle sorte de pain ? le veux-tu grillé au four ? fromage suisse ou cheddar? » (2012, p. 54). Cette mise en scène carnavalisante, rabaissant le sacré littéraire au niveau de l'alimentaire et du digestif, témoigne du statut du lieu, moins décor ou interface sociale qu'espace de consom-mation bêtement utilitaire. De même, lors de leur premier rendez-vous privé, hors de l'établissement de restauration, les deux personnages se voient dans un bar. Cela fait aussitôt l'objet d'une discussion :

T'es déjà venu Chez Véro ? [demande Tess]

- Euh... non, je pense pas.

- T'es plus du genre Café de la Voûte...

- En fait, je suis pas trop sorteux. Des fois, avec les gars de la shop, on va Chez Tonio.

- Et vous jasez du problème du temps chez Paul Valéry?

- Ça a jamais adonné, non. [...] » (2012, p. 71)

Les hiérarchies sont explicitées et niées aussitôt, ici; le capital culturel du Café de la Voûte est suggéré, accentué par son nom, le «Café » ${ }^{7}$ s'opposant au prosaïque bar ou à la taverne, tel Chez Véro, plus populaire. Cette question de capital culturel semble pourtant inopérante. Sébastien Daoust, plutôt misanthrope, se contente de visiter Chez Tonio avec ses collègues où, assurément, aucun capital culturel n'est mesurable, aucune question de légitimité littéraire, aucune valorisation des

\footnotetext{
${ }^{7}$ Je note au passage que, chez Blais, il existe peu de « cafés » au sens strict. Le terme, en fait, s'accroche plus aisément dans un certain contexte culturel. Le capital culturel induit au "café » de la voûte témoigne de ce phénomène. Je soutiens que des établissements comme le Tim Hortons, le restaurant Subway, les bars Chez Toy ou Chez Véro agissent comme des cafés : lieu de flânerie et d'interactions comme de restauration, ils sont des cafés sans en accepter - de nom - la tradition.
} 
savoirs spécialisés de la littérature n'agit. Le café et le bar ainsi décrits indiquent une condition essentielle de la littérature dans les romans de François Blais et que La Nuit des morts-vivants souligne à grands traits : à Grand-Mère - du moins dans celui de François Blais -, contrairement à Montréal, il n'existe aucune communauté lettrée, les règles factices qui organisent l'agir culturel en littérature y sont ou bien inexistantes, ou bien non fonctionnelles car sans autorité pour les régir.

J'ai évoqué plus tôt une représentation carnavalisante de la littérature. Tout indique en effet que l'œuvre de François Blais est traversée par une tentation carnavalesque, montrant un «monde carnavalesque par défaut» (2000, p. 13; l'auteur souligne), comme l'écrit Michel Biron. Ce carnaval s'avère moins un renversement de l'ordre et de la règle, de l'institution et du sérieux, que l'absence d'ordre et de règle, une friche culturelle dans laquelle les personnages lettrés paraîtraient sans repère s'ils n'étaient pas, justement, des personnages lettrés dans un roman. Car voilà le nœud de cette représentation de l'écrivain au café en société liminaire : sa communauté lettrée existe par le livre, elle est actualisée à chaque référence ironique - on rapproche la Lettre à un jeune romancier de Marc Fisher d'Esthétique et théorie du roman de Mikhail Bakhtine, par exemple -, alors que la diégèse ne présente que son désert culturel. L'absence d'interlocuteur au café de Grand-Mère, dès lors que cette absence est l'objet d'une représentation romanesque, dans un roman qui n'a pour sujet que le propre de la littérature et la fatuité de ses règles, jure avec l'évidence de cet interlocuteur au bout des pages, créant un espace dialogique ambigu, représentant la communitas carnavalesque pour la nier dans le même mouvement. 
La Nuit des morts-vivants explicite ce phénomène. Le roman met en place deux narrations, celle de Pavel et celle de Molie; tous deux ont été approchés par un homme mystérieux pour écrire chaque semaine quelques pages, contre rétribution. C'est dire qu'ils n'ont pas le profil de l'écrivain, plutôt lancés dans l'écriture par le choix contingent d'un «écrivain» en contrejour ${ }^{8}$. Le roman se construit donc sur ce dialogue entre, d'une part, un énonciataire implicitement lettré, montréalais induit ${ }^{9}$ et pouvant partager un certain nombre de connaissances avec les narrateurs et, d'autre part, une petite ville sans habitus intellectuel, où les cafés ne comptent que des lecteurs du Journal de Montréal. Entre ces deux pôles communicationnels, les narrateurs sont écartelés. On reconnaîtra la cabotine référence qui ouvre la narration de Pavel, traçant par là l'incipit du roman : «Longtemps, je me suis couché de bonne heure. Mais ce n'est plus possible depuis que je travaille de nuit pour Maintenance des Chutes à titre d'employé d'entretien (classe 2). » (2012, p. 5) Le détour-nement de l'incipit proustien représente assez bien la scène d'énonciation en place, entre un lecteur capable de décoder la référence littéraire et un univers incapable de l'accueillir : Proust devient ouvrier ! De même pour la seconde narratrice, Molie, qui mime par son écriture le monologue de Molly Bloom : « et là je viens de me relire du début et je me rends

\footnotetext{
8 J'ai développé cette notion, en abordant notamment l'œuvre de Blais, dans «En contre-jour. La représentation évanescente de l'écrivain dans le roman québécois contemporain » (2016).

${ }^{9}$ Je ne veux pas m'arrêter trop longuement sur ce point; toutefois, on peut noter que les références culturelles - liées aux pratiques culturelles plus précisément - prennent sans cesse en compte un énonciataire montréalais. Ainsi des descriptions géographiques des promenades de Molie, seconde narratrice; entre autres exemples, ce passage explicite : «Ici, le lecteur dira : “Oh, mais elle n'a qu'à rentrer en taxi, cette gourde”, ce qui prouve que le lecteur n'est pas sorti de Montréal souvent. » (2011, p. 27)
} 
bien compte que ça n'est pas génial mon idée d'imiter Molly Bloom j'ai du mal à me comprendre moi-même » (2012, p. 16). Dans cette structuration d'un savoir légitime mis en scène dans son échec, nous avons l'enjeu principal de la littérature chez Blais, et le café, le bar, n'en répète que l'évidence.

« Il y a à Grand-Mère un nombre ridiculement élevé de bars, tavernes, brasseries, cafés, clubs, bistros, etc. en regard du nombre d'habitants » (2011, p. 29), confie Pavel au moment de présenter son « nouveau passe-temps » : « s'asseoir au bar Chez Toy avec un livre, les fins d'après-midi, et [y] siroter longuement une bière » (2011, p. 29). Chez Toy accueille difficilement ce concierge lettré, qui consacre sa lecture à Middlemarch de George Eliot. D’ailleurs, la serveuse, Zoé, sur laquelle fantasme le narrateur, ne manque pas de demander si Monsieur George Eliot est un bon écrivain : «Grands dieux ! Voilà le genre de question que la Zoé idéale n'aurait jamais posée ! À n'importe qui d'autre [Pavel] aurait sans doute rétorqué que personne, pas même Jacques Demers, n'ignorait que George Eliot était une femme ». (2011, p. 47) La communauté du bar, la communauté de GrandMère que le bar métonymiquement met en scène, n'a pas à maîtriser un savoir lettré, la légitimité de ce savoir n’opère pas, sauf dans le discours des narrateurs, dépositaires de cette norme extérieure à la communauté qu'ils ne peuvent toutefois appliquer. Ainsi, faisant la cour à une caissière de l'épicerie Maxi, Pavel mesurera la valeur de la demoiselle à l'aune de la même norme inapplicable. Devant le laconique et peu stylisé message que la caissière lui adresse, en effet, Pavel jugera : « Décidément, Madame de Sévigné pouvait dormir tranquille. » (2011, p. 129)

Bars et cafés où traînent les écrivains de François Blais ne mettent qu'en saillie leur inadéquation avec leur communauté. 
Comme le Subway où Tess et Sébastien Daoust nouent leur premier contact, la séance de lecture de Middlemarch que Molie, à son tour, passe au Tim Hortons souligne l'exotisme de leur position culturelle: «j'ai lu une bonne centaine de pages de Middlemarch sans me faire écœurer c'est ça qui est cool dans les Tim on ne te pousse pas trop à consommer pour le prix d'un petit café tu payes ta place pour la journée » (2011, p. 103). Entre le style, qui mime encore confusément le monologue de Molly Bloom, le langage qui ne s'embarrasse guère d'un registre élevé, la référence littéraire raffinée et le lieu populaire sans lustre social où tout cela prend place, nous avons des suites de contrastes, des conflits normatifs. On peut dire alors que le café de Grand-Mère permet d'inscrire les narrations, aussi lettrées soient-elles, dans une communauté sociale qui les remet à leur place : on n'y parle pas du temps chez Paul Valéry, on n'y fabule pas dans un style proustien, on ne s'embarrasse pas des prestiges de café : le capital culturel de l'El Dorado chez Lessard ne fait aucun doute; ici, rien de tout ça : on y paye sa place, tout simplement, sans que celle-ci n'organise quelque hiérarchie sociale.

Nous avons ici, avec l'écrivain au café - qui toutefois n'est pas un écrivain -, ces « antagonismes de classes [souvent vécus au Québec] comme s'ils étaient modulés par le caractère universel et utopique de la culture carnavalesque » (Belleau, 1984 , p. 142). Ces antagonismes prennent la forme, comme l'énonce André Belleau, d'un rejet, d'une suspicion culturelle face à la culture légitime :

Je suis convaincu que la société québécoise, pour des raisons historiques, est demeurée jusqu'à nos jours profondément imprégnée par la culture populaire. La vision du monde qu'a le peuple s'oppose à celle qu'exprime la culture dite « officielle». 
Et elle se veut aussi totale, englobante que l'autre. D'une part, le langage unilatéral du pouvoir, de l'ordre, des salons, du snobisme, de l'autre, le langage comique, ambivalent du juron, du sacre, de la facétie, de la parodie, de la « grossièreté ». (1984, p. 142)

Dans cette dualité, on retrouve le "salon », mis aux côtés du snobisme, de l'ordre, du pouvoir; contre le salon et ses hiérarchies apparaissent la farce, le grossier et, pourrait-on dire, le café. Le café représente, chez Blais du moins, cette absence de hiérarchie, où les langages - comique ou sérieux, englobant ou unilatéral - se juxtaposent et voisinent sans se dominer. Le caractère utopique de cette culture carnavalesque brille fortement, car on sait bien qu'un monde où l'ordre n'agit plus ne peut qu'être fantasmé. En effet, les normes, quand bien même elles ne font pas l'objet d'un consensus, quand bien même elles rentrent en conflit les unes avec les autres, agissent continuellement. Une dernière scène de café en cristallise l'esprit.

Pavel participe à un "party de Noël » dans un bar de Grand-Mère; la fraternité des hommes, tous employés à Maintenance des Chutes, donne à la soirée un fort sentiment de cohésion sociale. Néanmoins, Pavel ne peut simplement se fondre à la solidarité en place : «Chaque fois qu'il se retrouvait entouré d'imbéciles, Pavel aimait bien, par coquetterie, les coiffer au poteau sur leur propre terrain » (2011, p.138). Le protagoniste décoche alors un mot d'esprit qui, par sa subtilité moquerie ironique que ses confrères ne savent décoder - «lui valut un franc succès » (2011, p. 138). Pavel - qui prend le parti de se raconter à la troisième personne, dans un style soutenu, parfois fleuri - juge les hommes qui l'entourent dans le café, comme il juge tous ceux qu'il croise Chez Toy. Ses jugements, son 
mépris, sa hauteur restent toutefois sous cape; le plus souvent, il ne partage ses sentiments culturels qu'avec le lecteur ou encore, comme ici, se terrant derrière quelques fleurs rhétoriques. La norme et le pouvoir qu'elle confère à celui qui la maîtrise ne s'aboliraient donc pas dans le café, même dans un café de GrandMère ? En vérité, il faut noter que, maîtrisant cette norme, Pavel ne peut toutefois l'appliquer, la faire agir dans sa communauté; Montréal, la scène culturelle, le savoir lettré, le livre seuls en permettent l'expression. Sans cette scénographie narrative, sans la complicité avec une communauté interprétative reconnaissant une valeur à son énoncé, Pavel ne peut mettre en place son mépris, car dans son univers, le lettré ne peut qu'avoir tort - ainsi en est-il du jeune employé de Maintenance des Chutes, J-F, étudiant à la maîtrise en littérature, qui est un « désastre comme employé d'entretien » (2011, p. 18). Lorsque Pavel choisit de se retirer dans la fête, de trouver un lieu paisible où lire dans le bar, il marque bien que "si on surprenait quelqu'un en train de bouquiner dans un party de Maintenance des Chutes, on érigerait sans doute un bûcher » (2011, p. 141). Grand-Mère, le café et sa communauté ont leurs propres règles, mais celles-ci ne trouvent une forme qu'ironisée, édulcorée, prennent l'apparence d'une ignorance et d'une indifférence, donnant à la norme du narrateur tout son lustre : sa force de lucidité et de conscience semble alors évidente. Pavel se retire effectivement dans la fête, il grimpe à la mezzanine du bar où, en surplomb des fêtards, il boit doucement une bière en se plongeant dans un livre.

À la vérité, le café symbolise sans doute une sorte d'espace carnavalesque chez François Blais; dans cet espace, la culture lettrée se trouve déplacée. Mais ce déplacement n'agit pas tel un renversement; il permet plutôt de mieux mettre en scène le lettré liminaire, les tensions qui le traversent dans un monde ouvrier 
où sa norme et sa connaissance n'ont guère de sens. Cette représentation du café fait un aller-retour, car si La nuit des morts-vivants raconte des lettrés ironisant sur la «bêtise ambiante », sur l'inculture, Document 1 ironise sur l'habitus littéraire, ses marchés symboliques et la facticité de ses règles. Le café devient une sorte de frontière, moins lieu de cohésion sociale que de partage - même si cela parfois ressemble à une confrontation. L'écrivain au café de Grand-Mère ne peut y être dans son élément. Sa liminarité, toutefois, ne saura qui accuser de la communauté interprétative ou de la communauté ouvrière.

\section{Sans adresse : le café chez Mylène Bouchard}

Le café est un espace de dialogue : ce constat paraît appelé dès l'analyse de l'œuvre de Lessard, où l'appartenance paradoxale de l'écrivain à sa culture se jouait. Un phénomène parent s'observe dans l'œuvre de Blais, la communauté lettrée du livre y dialoguant avec la communauté ouvrière de la petite ville. Dans les deux cas, mais fort différemment, la norme littéraire et l'abstraction culturelle y perdent leur efficace au profit d'une incarnation de la vie culturelle, laquelle ne peut que mettre en place le conflit des normes. Dans cet espace de dialogue, le langage unilatéral du pouvoir est inaudible.

Le café, chez Mylène Bouchard, propose autre chose, me semble-t-il. Ma démonstration, rapide, me permettra pourtant d'arriver à une conclusion. La Garçonnière présente plusieurs cafés, tous carrefours, moments de basculements, intersections : le Café Typo dans le film culte des protagonistes, The Pillow Book, où les amoureux se rencontrent, le Café Ciné-lumière où Hubert, écrivain, rencontre Richard Desjardins et apprend de sa bouche 
les secrets sur l'amour, le Café suspendu, surtout, où les protagonistes se rencontrent tous les matins, où ils mettent fin à leur relation également. Se condamnant à l'exil pour surmonter sa peine d'amour, Hubert, en ce sens, regrette surtout ce café et ce qu'il signifiait dans sa vie :

Par-dessus tout, il peinerait à laisser derrière lui le Café Suspendu. Oui. Malgré la mort de l'homme, malgré la scène de la rupture, l'endroit demeurait un impératif de tous les instants. Ce lieu représentait cette époque significative. Il était parti de Péribonka alors qu'il ne connaissait pratiquement rien sur luimême, encore un enfant, à dix-sept ans. Il faisait cavalier seul. [...] Une époque riche en rencontres, en apprentissages, en rêves. Ce café, c'était cette escale, précisément. (2009, p. 145)

Le café forme la communauté autour d'Hubert; il se substitue à ce monde plein de Péribonka qu'il venait de quitter. Il faut dire que le concept du café « était tout à fait hospitalier » :

$\mathrm{Au}$ Café Suspendu[, le client] était invité à suspendre un café au moment de régler la note, payant ainsi un café consommé puis un de plus. De cette façon, n'importe qui, à toute heure du jour, pouvait s'arrêter au café et demander au serveur : «Auriez-vous un café suspendu par hasard ? » Si la réponse s'avérait positive, le passant buvait un café et repartait, le pas léger. [...] Le propriétaire en avait profité une fois, à Prague. U Zavesenheocafe, Prague. Et plusieurs années plus tard, vadrouillant à Montréal, il avait ouvert son lieu sympathique sous la même appellation. (2009, p. 57)

Le café agit alors comme un lieu de passage et de partage; en ce sens, il détermine les déplacements de l'écrivain: « Naturellement, parce qu'il y avait là un Café Suspendu, il avait opté pour Prague. » (2009, p. 145) Le café s'avère donc détaché du lieu; il transite, se déplace, existe à divers endroits. S'il reproduit, à Montréal, la communauté perdue de Péribonka, il déplace, à Prague, la communauté abandonnée à Montréal. Il me 
paraît difficile de ne pas lier cette connotation du café au discours moderniste qui traverse le roman de Mylène Bouchard. Dans sa forme et dans son propos, en effet, se devine dans $L a$ garçonnière une confiance moderne envers le matériau littéraire, dont la représentation d'Hubert en tant qu'écrivain est symptomatique : sujet lyrique et passionné, son rapport à la création et au succès semble dénué de complexes et d'obstacles. Le café comme lieu commun de la littérature est alors accepté, avec sa cohérence esthétique et sa cohésion sociale. Ainsi, lorsque Hubert s'assoit près de la fenêtre du Café Suspendu, la narration ne manque pas de noter : «Un écrivain adore avoir vue sur une scène extérieure où chaque individu est porteur de littérature. » (2009, p.117) Depuis le café, Hubert perçoit la littérature dans tout; le lieu devient bien davantage qu'un espace social, il représente le regard même de la littérature, son détachement de la rue avec vue sur celle-ci. On pourrait dire qu'il est alors, à l'instar d'un discours romantique sur la littérature, sans adresse, mais ici selon deux significations : d'une part, le café n'est pas localisé, il représente bien davantage un esprit partagé de Montréal à Prague, une communauté délocalisée dans sa multiple localisation; d'autre part, il est sans adresse par sa représentation de l'énoncé littéraire, non adressé: «La littérature est le règne de l'écriture, de la parole qui circule en dehors de toute relation d'adresse déterminée ", écrit en ce sens le philosophe Jacques Rancière :

Cette parole muette, disait Platon, s'en va rouler à droite et à gauche sans savoir à qui il ne convient pas de parler. Il en va ainsi pour cette littérature qui ne s'adresse plus à aucune audience spécifique, partageant une même position dans l'ordre social et tirant de cet éthos des règles d'interprétation et des modes de sensibilité ordonnés. (2007, p. 21) 
On peut dire effectivement que la littérature est marquée dans ce roman par l'esprit libre - mais non décalé - de la littérature : la littérature apparaît dans chaque individu, mais elle n'est adressée à aucun; l'œuvre, détachée de tout contexte, survient tout simplement comme un café suspendu, destiné à personne en particulier. Ainsi, Hubert publie ses premières œuvres une fois exilé à Prague; son succès international porte en germe une foi envers ce langage universel, sans audience spécifique. Cette foi prend une autre forme également, celle d'une anecdote initiatique pour l'écrivain. « Il faudrait raconter que les mots ont eu raison sur la vie de ce buveur de café, amant des cafés, abonné du Café Suspendu. » (2009, p. 34), lit-on au début du roman. Le Café Suspendu semble ainsi, dès l'amorce, hanté par un pouvoir particulier des mots. «Un dernier café et j'y vais » (2009, p. 35), se serait exclamé l'homme dans le Café Suspendu. Et ce fut en effet la dernière chose que l'homme but, frappé aussitôt par une voiture, dans la rue.

Cette scène confirme d'une certaine manière le discours sur la littérature que le café recouvre; un discours qui dit la présence de la littérature partout et l'usage de la littérature dans un monde où les mots peuvent encore avoir quelque importance. Si la scène du mort a un caractère initiatique pour Hubert, c'est parce qu'elle lui fait abandonner la photographie au profit de l'écriture. Entre un réel capté par la machine et un réel influé par le langage - car il ne fait pas de doute pour Hubert que le « dernier café » prononcé a performé la mort du pauvre homme - le choix semble simple.

La Garçonnière rejoue donc le topos du café; le lieu d'une fête et d'une effectivité de la littérature, d'une cohésion - d'un sens - des lettres dans le monde, d'une cohérence - universelle - 
de la littérature dans le livre. Il n'y a qu'un pas pour voir ici apparaître un langage du pouvoir, qui, on l'a vu, restait en retrait des cafés chez Lessard et chez Blais. L'idéologie d'une littérature romantique y semble effectivement lourde. Gardons-nous, pour le moment, de franchir ce pas.

\section{Trois cafés : interactions de la littérature}

Entre le café et l'écrivain, il existe une sorte de nécessité, à énoncer sous forme d'évidence: l'écrivain doit forcément écrire quelque part. Cette nécessaire localisation appelle une interaction problématique, car être planté dans un lieu, c'est surtout évoluer dans une communauté. Comme l'écrit François Paré, «il y a eu meurtre de l'espace dans un certain nombre de grandes cultures; ce geste mémorial a permis et permet toujours rituellement d'inscrire ces cultures dans une pure problématique du temps et ainsi dans ce qu'on pourrait appeler un discours de l'éternité » (2001 [1992], p.115-116). Tuer l'espace consiste à se délocaliser, à récuser la nécessité du lieu dont parle Maingueneau. Représenter l'écrivain au café, au contraire, souligne sa localisation, réifie l'espace, car si les lieux fermés appellent les interférences du quotidien - ainsi de la chambre, de la cabane reculée, de la cellule -, ils ne rendent pas nécessaire la constante interaction avec une communauté, sous forme d'idéologie ou d'individus. Le café est carrefour, lieu non vierge, saturé de connotations et de discours. L'écrivain y sera assurément confronté.

De là, je ne peux qu'arriver à un premier constat qui départagera les œuvres de mon corpus. La confrontation, pour ainsi dire, n'agit pas de la même manière partout. Chez Lessard comme chez Blais, il a été facile de remarquer que la littérature - comme 
discours, valeurs, langage - risquait de s'opposer aux discours de la société. Ce risque appelait diverses représentations de l'écrivain au café, tantôt s'y intégrant, tantôt s'y désintégrant, parfois en conflit de valeurs, parfois en fête, acceptant de laisser la littérature au vestiaire. Ce trait se trouve au centre des œuvres de Lessard et de Blais et, en ce sens, la même image a été utilisée pour décrire leurs cafés dans leur médiation de la littérature : frontière, portail, bref, lieu de va-et-vient, de dialogue. Mais il semble que le discours sur la littérature que porte La Garçonnière de Mylène Bouchard ne puisse laisser cours au même dialogisme. En effet, chez Bouchard, la littérature se trouve solidement érigée sur une sorte de discours romantique; l'idéal de cette littérature est marqué par un schématisme dans sa représentation dont le café partage les traits. Ainsi, les lectures, l'écriture, le milieu de l'édition sont l'objet d'incarnations dans les œuvres de Lessard et de Blais - Elliot, Nabokov, le geste d'écrire, tout cela est intégré à la forme du récit , mais chez Bouchard, la référence se colle difficilement, agit plutôt par son seul capital, sa pure valeur culturelle. L'écrivain du roman, Hubert, publie dès son exil à Prague et la narration joue de l'ellipse : on se contente de nous mentionner que son succès est grand et que les éditeurs se l'arrachent. Le café explicite cet idéalisme en n'accueillant que des personnages issus du milieu culturel : Hubert y rencontre Mara, animatrice culturelle à la radio, de même pour Richard Desjardins. Autrement, les interactions servent strictement à faire de la littérature, à en confirmer les fondements - ainsi de l'homme qui meurt par excès de langage. Il n'y a donc qu'un seul milieu au cœur du café, et ce milieu est, comme j'ai voulu le montrer, mouvant, sans adresse, planté plutôt dans la " grande culture » et la «problématique du temps» que dans le prosaïsme de la localisation. On peut dire, en fait, que l'absence de grandiloquence et le cynisme permettent aux œuvres de Lessard et de Blais de 
représenter le lieu conflictuel du café pour penser à de nouveaux frais les modalités d'intégration de la littérature au reste de la société. Il faut d'une certaine manière accepter la déréliction des hiérarchies classiques, réaliser la facticité de la chose littéraire audelà de son idéal pour en représenter les conflits; le café et ses conflits, dans ces œuvres, disent beaucoup en vérité le maintien d'une interaction - vécue dans sa nécessité - entre le monde et les lettres.

De là, un deuxième constat doit être tracé. Il a été dit que le lieu commun du café n'appartient guère à l'histoire littéraire québécoise; il convenait de voir comment cette tradition d'un autre a été adaptée, modélisée, travaillée. Situés à Prague, à Lisbonne, à Paris, les cafés que nous avons visités sont souvent décalés, inscrits diégétiquement au cœur de la tradition européenne à laquelle le café appartient en propre. Les fuites de Lessard ou l'exil d'Hubert s'incarnent ainsi dans une représentation conventionnée de l'Europe littéraire et adaptent par là sa mythique. À l'inverse, les bars et cafés de Grand-Mère sur-jouent leur éloignement - du centre culturel comme de la tradition. Rien ne semble moins culturel que ces lieux où aucun "savoir légitime», aucun sujet symbolique n'agit. En fait, seul l'El Dorado occupe dans le jeu des représentations la place du café typique de la littérature incarné à Montréal. Le décalage culturel dont il est l'objet, l'éviction inaugurale de l'écrivain qu'il accueille sous forme de métalepse indique toutefois sa difficile incarnation.

André Belleau remarquait, dans son Romancier fictif, que la représentation de la littérature dans les années 1940-1950 « donn[ai]t à voir la littérature détachée de toutes conditions de production [en] traduisant par là même une conception nettement idéaliste » (1999, p.133). Cet idéalisme s'avère en saillie dans 
La Garçonnière : en détachant l'écrivain du lieu, en faisant de ce lieu - ici, le café - un non-lieu, il révèle cette conception idéaliste. On peut dire que l'attention cynique, lourdement ironisée, que François Blais accorde aux moyens de productions littéraires - les conseils des arts, les éditeurs - apparaît dans le lieu même, car le café, le restaurant, le bar y semblent plus qu'ailleurs sans valeur d'éternité, pur espace, pure localisation. La littérature, vraiment, n'y est que conditions de production. Entre ces deux extrémités, il y aurait l'El Dorado de Patrice Lessard, un lieu un peu inaccessible son nom paraît révélateur - lieu où la culture concrète et le symbole de la culture coexistent dans l'incohérence, dans le quiproquo, dans la mésentente, comme si la seule coexistence possible en littérature ne pouvait qu'être faite de négativité. Le café nous aurait alors enseigné ceci sur le discours sur la littérature : voilà moins le lieu d'une cohésion sociale que d'une interaction, devenue rare, entre le discours littéraire et les autres discours. On aura beau parler de Carnaval, espérer - et tout un discours sur la littérature y invite, appelant une totalité plurivoque, un « égalitarisme radical » entre les mots (Rancière, 2007, p. 19) - une littérature débarrassée des codes et normes, des hauteurs et facticités, ce n'est pas le café qui incarne tout cela. Le café ne trouve plus guère de salon auquel s'opposer. Il n'est plus qu'un lieu pur d'interactions. Interactions avec la tradition même du café littéraire, avec les discours qui le traversent, entre la vie et la culture qui l'organisent, entre les codes, jamais les mêmes, du Journal de Montréal jusqu'à un livre de Nabokov. Ces interactions montrent évidemment le caractère bringuebalant de la chose - la littérature - qui paraît un peu fausse, simple institution, coquille vide se survivant comme " un fantôme » pour reprendre l'expression de Pierre Nepveu (1988, p. 13). Les écrivains au café, pour peu qu'ils ne soient pas tentés par 
l'idéalisme, interagissent donc dans la crainte que ce soit le dernier justement; le dernier café de la littérature.

\section{Bibliographie}

BÉLANGER, David. (2016), «En contre-jour. La représentation évanescente de l'écrivain dans le roman québécois contemporain ", Arborescence, no 5, p. 54-71, <https://www.erudit.org/revue/arbo/2016/v/n6/1037504 ar.pdf>.

BELLEAU, André. (1984), Y a-t-il un intellectuel dans la salle?, Montréal, Les Éditions Primeur, coll. «L'échiquier ».

-. (1999 [1980]), Le Romancier fictif. Essai sur la représentation de l'écrivain dans le roman québécois, Québec, Nota bene, coll. «Visée critique».

Biron, Michel. (2000), L'Absence du maître. Saint-Denys Garneau, Ferron, Ducharme, Montréal, Les Presses de l'Université de Montréal, coll. « Socius ».

—. (2015), Saint-Denys Garneau, Montréal, Boréal.

BLAIS, François. (2011), La Nuit des morts-vivants, Québec, L'instant même.

—. (2012), Document 1, Québec, L'instant même.

BouchARD, Mylène (2009), La Garçonnière, Chicoutimi, La Peuplade.

CôTÉ-FOURNIER, Laurence. (2016), «Les éditions Rodrigol : un formalisme du commun », Études françaises, vol. 52, no 2, p. 29-46.

DANTIN, Louis. (1997 [1904]), Émile Nelligan et son oeuvre, édition critique établie par Réjean Robidoux, Montréal, Les 
Presses de l’Université de Montréal, coll. «Bibliothèque du Nouveau Monde ».

LAISNEY, Vincent. (2010), "Cénacles et cafés littéraires : deux socialités antagonistes », Revue d'histoire littéraire de la France, no 3, vol. 110, <https://www.cairn.info/revue-dhistoire-litteraire-de-la-france-2010-3-page-563.htm\#pa3>.

LESSARD, Patrice. (2009), Je suis Sébastien Chevalier, Montréal, Rodrigol.

-. (2012), «Comme des Hell's », dans Printemps spécial, Montréal, Héliotrope, p. 88-96.

-. (2014), L'Enterrement de la sardine, Montréal, Héliotrope.

Maingueneau, Dominique. (2006), Contre Saint-Proust ou la fin de la littérature, Paris, Belin.

NePveu, Pierre. (1999 [1988]), L'Écologie du réel. Mort et naissance de la littérature québécoise contemporaine, Montréal, Boréal, coll. « Compact ».

PARÉ, François. (2001 [1992]), Les Littératures de l'exiguïté, Ottawa, Le Nordir, coll. "Bibliothèque canadiennefrançaise ».

RANCIÈre, Jacques. (2007), Politique de la littérature, Paris, Galilée, coll. « La philosophie en effet ».

\section{Résumé}

Cet article analyse la représentation de l'écrivain au café dans le roman québécois contemporain. Pour ce faire, l'œuvre de trois auteurs est auscultée: Patrice Lessard Ue suis Sébastien Chevalier, L'Enterrement de la sardine), François Blais (La Nuit des morts-vivants, Document 1) et Mylène Bouchard 
(La Garçonnière). Le postulat de base guidant cette étude est que, dans la littérature contemporaine, le lieu commun du café d'écrivain est transformé et devient le lieu de l'interaction et de la confrontation de la littérature avec les autres discours sociaux.

\begin{abstract}
This article intends to observe the representation of writer in café in Contemporary Quebec literature. For this matter, books of Patrice Lessard Ue suis Sébastien Chevalier, L'Enterrement de la sardine), François Blais (La Nuit des morts-vivants, Document 1) and Mylène Bouchard (La Garçonnière) are analyzed.
\end{abstract}

